\title{
Applying Standard JPEG 2000 Part One on Image Compression
}

Maha Abdul Rahman Hasso

College of Computer Sciences and Mathematics

University of Mosul, Mosul, Iraq

Received on: 18/03/2013

Accepted on: 24/06/2013

\begin{abstract}
In this paper, has been proposed Algorithm for standard JPEG2000 part one for image compression. The proposed Algorithm was executed by using MATLAB7.11 environment, applied these algorithm on the gray and color images for type of the images natural, medical, Graphics images and remote sensing. Dependence on the Peak Signal-to-Noise Ratio (PSNR) for comparing the result of the proposed Algorithm by using the Daubechies filters 5/3 tap filter and 9/7 tap filter Biothogonal, Another comparison is held concerning the obtained results of the algorithm of ModJPEG and Color-SPECK. Proved the processing results Efficiency performance of proposed Algorithm.
\end{abstract}

Keywords: JPEG2000 part one, convention, Field Programmingable Gate Array.

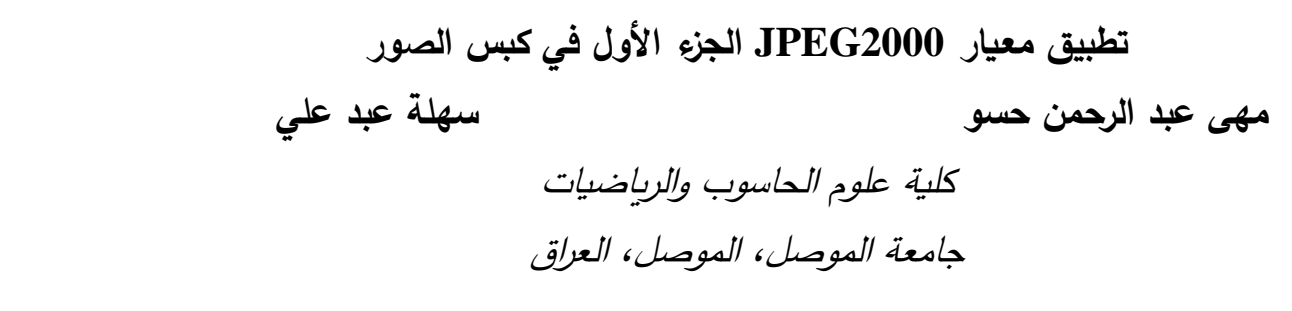

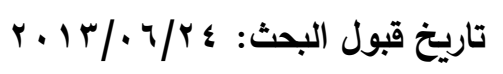

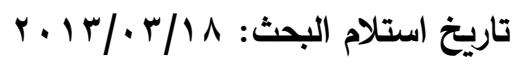

الملخص

تم في هذا العمل اقتراح خوارزمية لمعيار JPEG2000 الجزء الأول لكبس الصور. خوارزمية الكبس المقترحة تم تتفيذها باستخدام بيئة MATLAB7.11، طبقت هذه الخوارزمية على الصور الرمادية والملونة لأنواع من الصور الطبيعية والطبية والصور الرسومية وصور الاستثعار عن بعد. وتم اعتماد مقياس نسبة قمة الاشارة الى الضوضاء Peak Signal-to-Noise Ratio (PSNR) لمقارنة نتائج الخوارزمية المقترحة باستخدام مرشحي دبجي ثنائي التعامد 7/9 و 5/3 ومقارنتها مع نتائج خوارزمية ModJPEG و Color-SPECK المستحصلة. اثبتت النتائج العملية كفاية اداء الخوارزمية المقترحة مقارنة بالخوارزميات. الكلمات المفتاحية: JPEG2000 part one ، الالتفاف الرياضي، مصفوفة رقاقة البرمجة الحقلية. 1

يعد كبس الصور موضوعا مهما في العالم الرقمي وفي التطبيقات الاكاديمية والصناعية والتجارية سواء

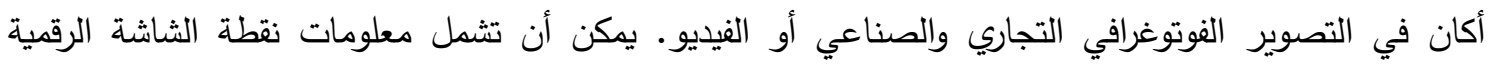
كميات كبيرة من البيانات إلى حد كبير ، ادارة مثل هذه البيانات يتطلب حملا زائداً للعمليات الحسابية 
المعقدة والخزن ومعالجة البيانات، ان سرعة الوصول المثالية لأوساط الخزن يتتاسب عكسيا مع السعة، لذا فإن كبس البيانات من المهام التي تحسن السعة [1] وتساعد في تقليل مساحة الخزن المطلوبة وهو ما يكافئ زيادة سعة لونة أوساط الخزن وتحسين سعة الإرسال، لذا فان تطوير تقانات الكبس الكفوءة مازال مستمرا من أجل تحدي تصميم

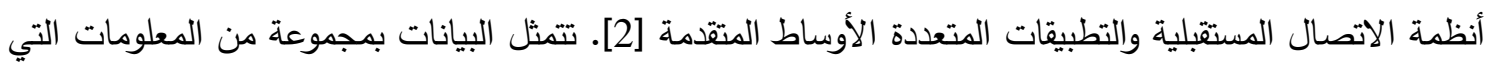
هي في كثير من الأحيان تكون متكررة. المعلومات وهي جزء البيانات الذي يجب أن يبقى بشكل دائم في شكله

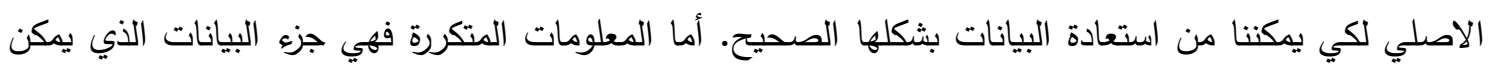

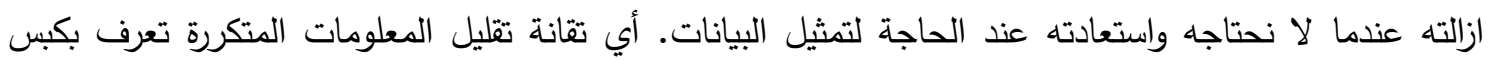
البيانات (Data Compression)، المعلومات المتكررة في تمثيل البيانات تقلل بطريقة بحيث يمكن بعد ذلك اعادة ادخالها لاستعادة البيانات الأصلية، والتي تعرف بفك كبس البيانات (Data Decompression) [2]. احد انواع خوارزميات الكبس هو JPEG2000 وهو معيار جديد لكبس الصور طور تحت رعاية (Photographic Experts Group) (Joint JPEG المعروف باسم لجنة) ISO/IEC JTCI/SC29/wG1 [3]. هذا المعيار ينحدر بشكل جذري من سلفه المعروف أكثر JPEG، ففي JPEG يتم استخدام تحويل جيب التمام المتقطع ( Discrete Cosine Transform DCT) في حين يكون استخدام الترميز الحسابي والتقريب المتتالي اختياريا فيها، أما في JPEG2000 فاستخدم تحويل المويجة المتقعع (Discrete Wavelet (Transform DWT الكبس دون فقدان [4]. نظرا لكون معيار JPEG فيه عدة عيوب في بعض من التطبيقات الجديدة مثل الانترنت والاتصالات اللاسلكية النقالة أو التصوير الطبي لذلك بدأت لجنة JPEG في 1996 بتحري امكانيات تكوين معيار كبس جديد وهو JPEG2000، أن تكوين هذا المعيار ليس فقط لتحقيق أعلى نسبة كبس بكفاءة مقارنة مع

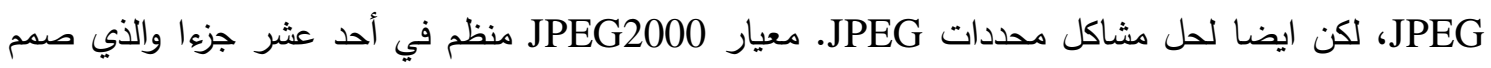
لتطبيقات مختلفة [5]، حيث زود بمجموعة من الميزات ذات الأهمية الحيوية لعديد من التطبيقات [2].

\section{2. نموذج لنظام JPEG2000 CODEC}

يتكون نموذج CODEC المقترح الخوارزمية معيار JPEG2000 الجزء الأول من نظام الترميز ونظام فك الترميز. الصورة المراد كبسها قد تكون ملونة أو رمادية، الصورة الرمادية تتكون من مكون لوني واحد بينما الصورة

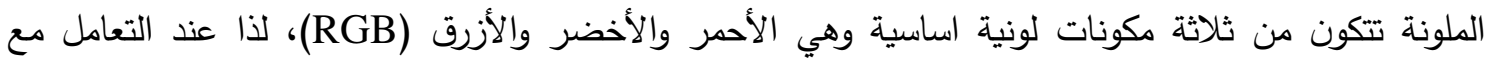
الصورة الملونة فإن كل مكون لوني فيها يمكن التعامل معه واعتباره صورة رمادية لكن بعد تحويل الصورة الملونة إلى فضاء YUV ومن ثم تجزئته الى ثلاثة مكونات وهي Y و U V V، Vيث يتم كبس كل مكون بصورة مستقلة، ومن ثم تحويله الى صيغة نصية وخزنه لإمكانية نقله واستلامه من نظام فك الترميز الذي يمكنه من استرجاعها

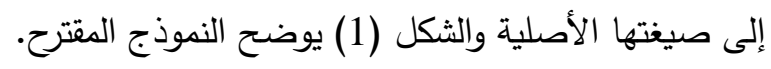




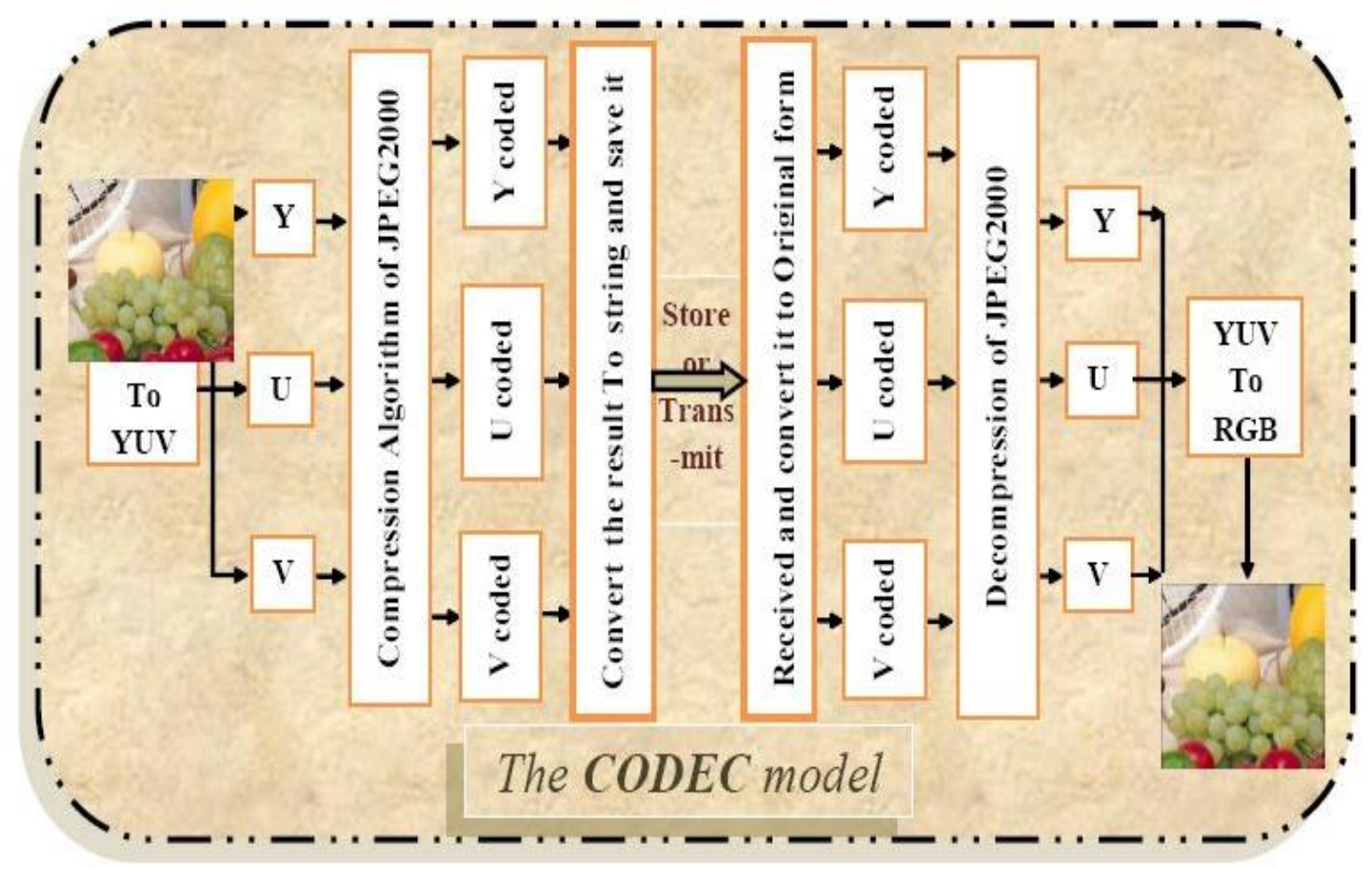

الشكل(1): نموذج CODEC المقترح لخوارزمية JPEG2000.

3PEG2000 Part1 3نظم ترميز

يقسم نظام الكبس المتكامل ببساطة إلى ثلاث مراحل، (1) المعالجة الابتدائية للصورة، (2) الكبس و (3)

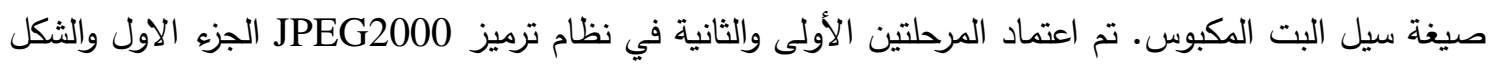
(a)(a) يوضح الرسم التخطيطي لوظائف نظام ترميز المعيار والثكل (b) يوضح مسار البيانات النظام الكبس.

4. تطبيق الخوارزمية المقترحة

يوضح الثكل (3) الخطوات الرئيسة الخوارزمية الكبس المقترحة لمعيار JPEG2000 الكيس الصور التي طبقت في بيئة Matlab. كما هو موضح من الثكل أن الخوارزمية المقترحة تتكون من نظام الترميز وفك الترميز . 1.4 في جهة المرمز Encoder Side: في البداية يتم اولا قراءة الصورة، يتم بعدها اجراء عملية التحويل اللوني

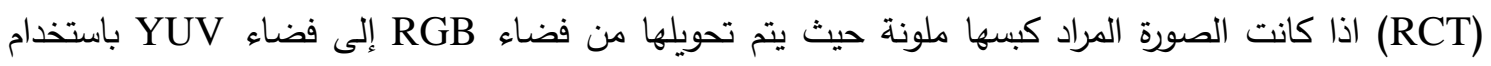
المعادلات الموضحة أدناه، بعد عملية تحويل الصورة الملونة نحصل على ثلاثة مكونات لونية أو مصفوفات وهي لهوني $. \mathrm{V}, \mathrm{Y}$ ،U

$Y=\operatorname{round}((R+2 * G+B) / 4)$

$\mathbf{U}=\mathbf{R}-\mathbf{G}$

V=B-G 


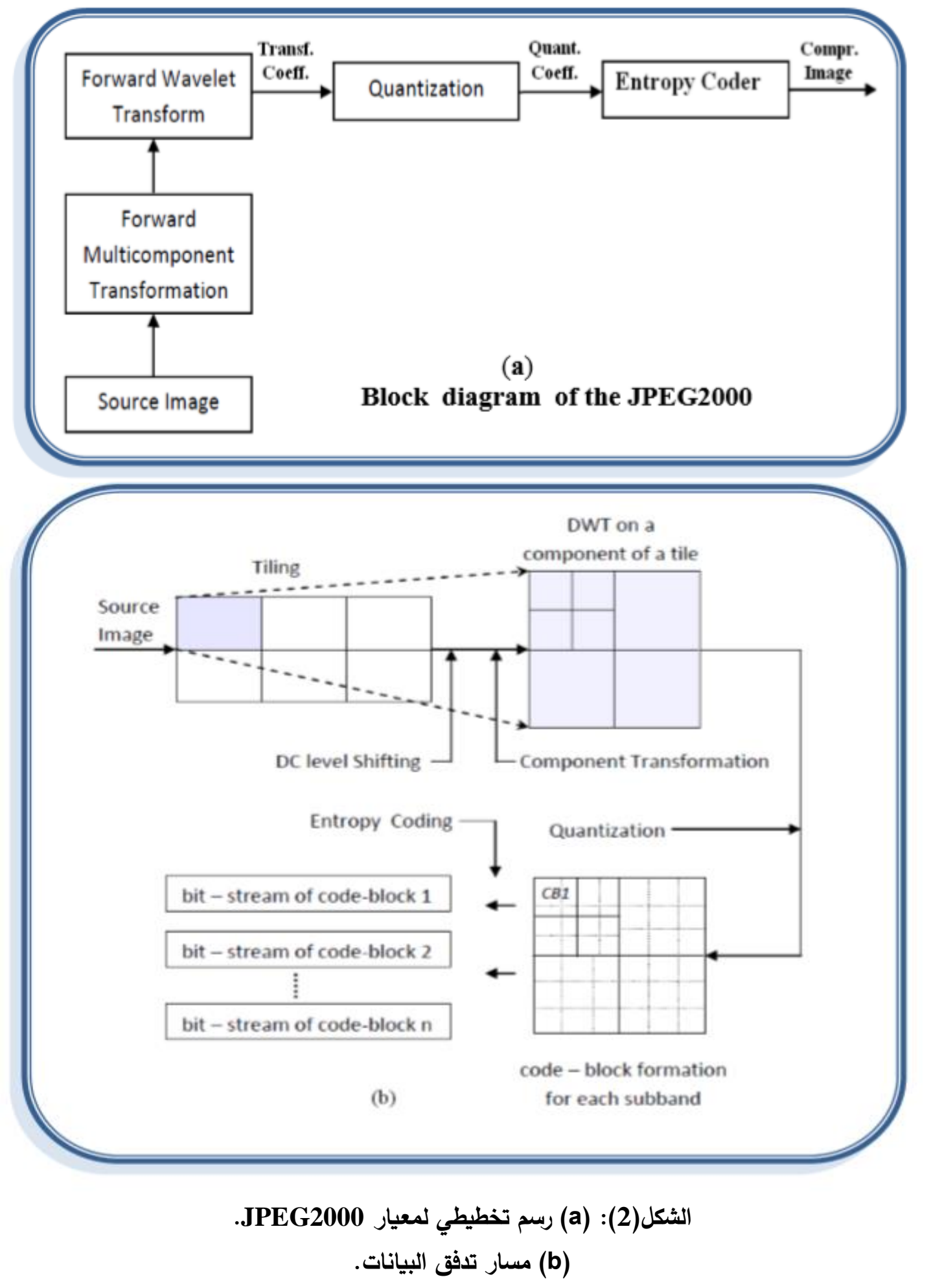




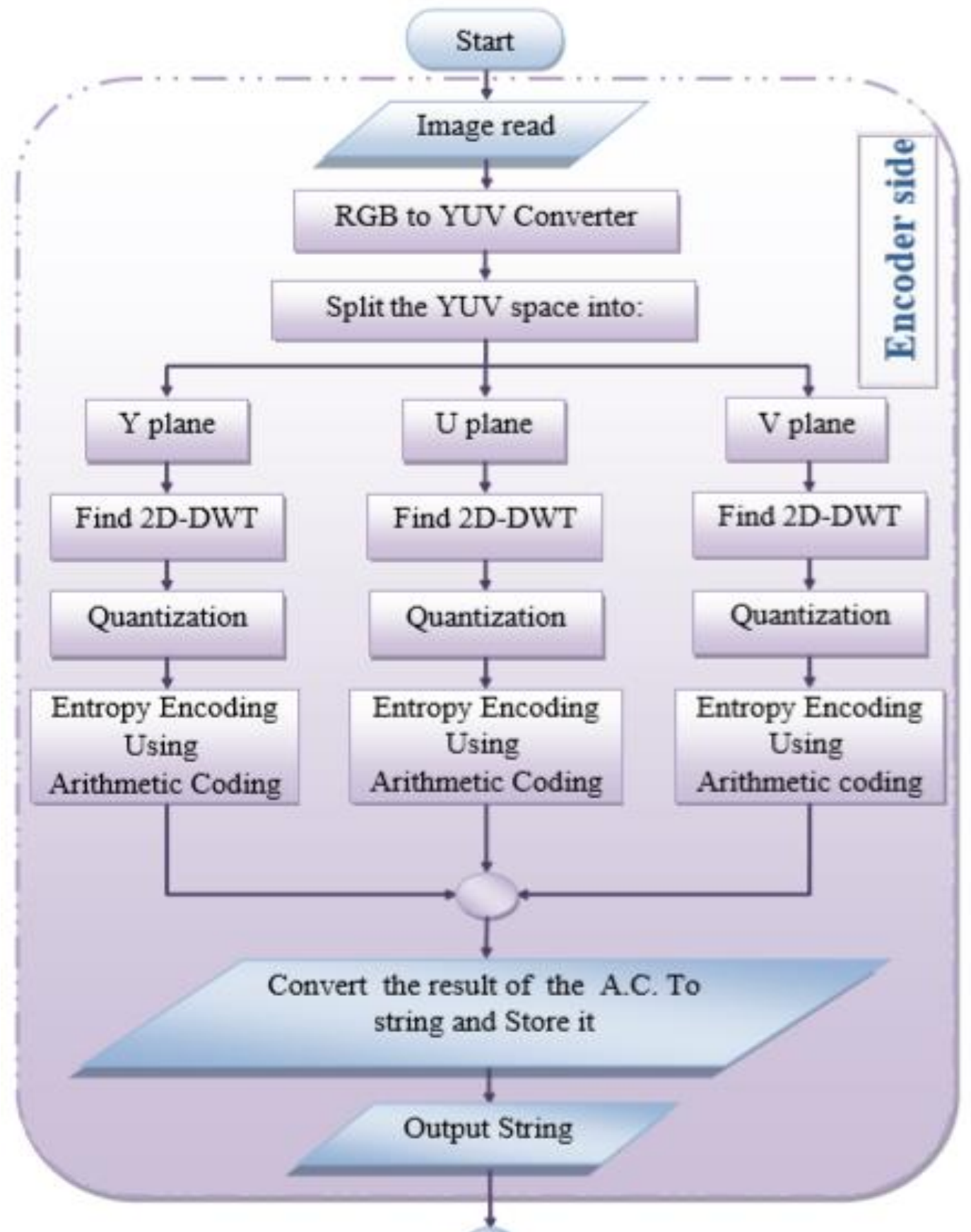

A

(a) 


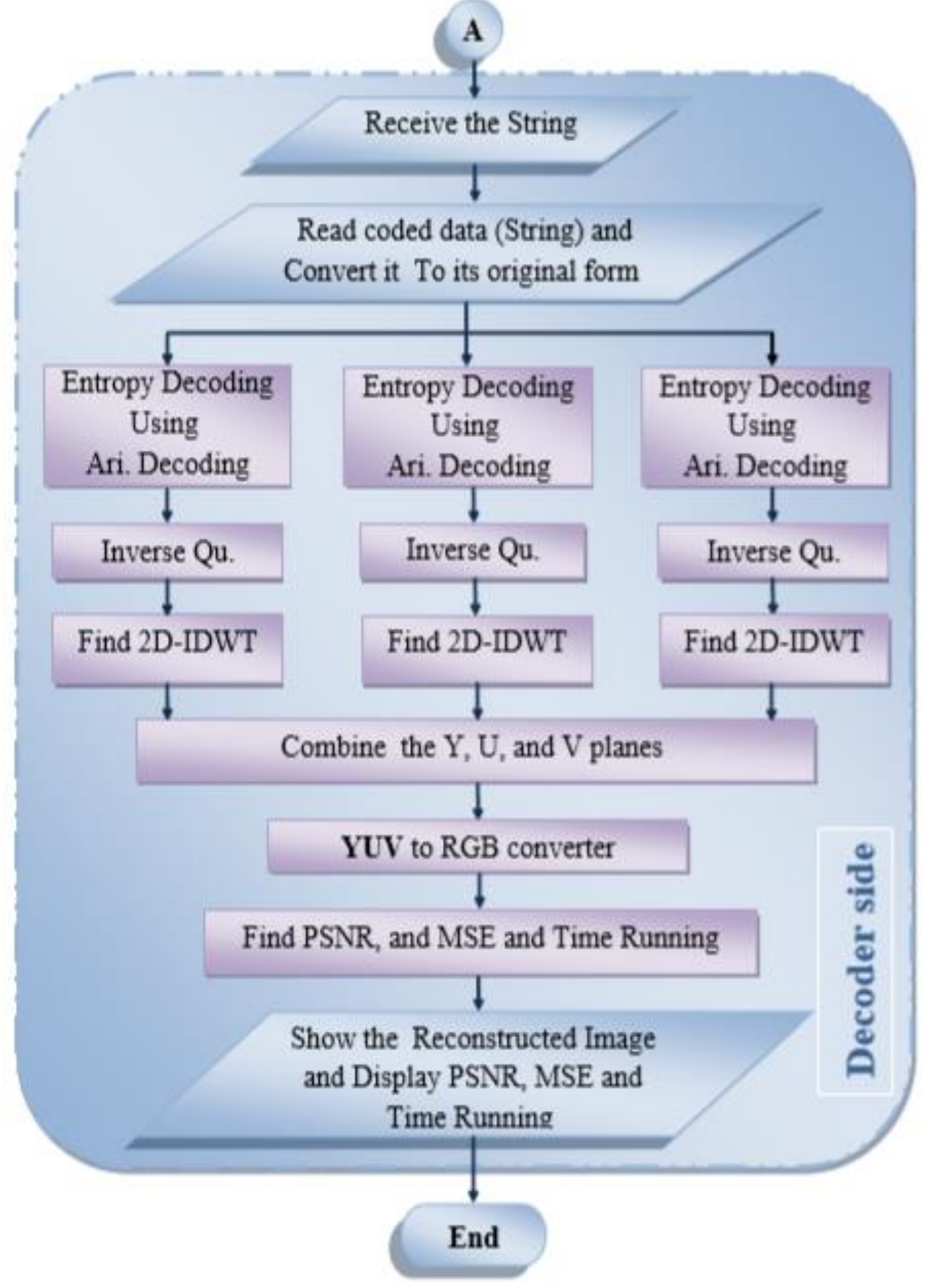

(b)

الشكل(3) (a) و(b) المخطط الانسيابي للبرنامج الرئيس لخوارزمية الكبس المقترحة لـ JPEG2000.

بعد اجراء التحويل اللوني في حالة الصور الملونة، يتم بعدها تطبيق خطوات خوارزمية الكبس المقترحة .JPEG2000」

1.1.4 الخطوة الأولى لخوارزمية الكبس: تطبيق التحويل المويجي المتقطع ذات بعدين (2D-DWT)، حيث

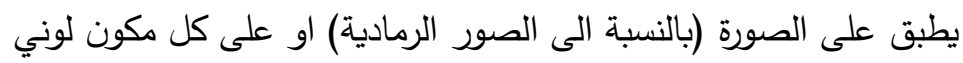


بالنسبة إلى الصور الملونة YUV) بصورة مستقلة، حيث يُحلل الصورة او المكونات اللونية في الصورة الى حزم فرعية (sub bands)، باستخدام مرشحات التمرير العالي والواطئ. ان تحويل المويجة ذات بعدين والذي ينجز بخطوتين: في الخطوة الأولى يطبق تحويل المويجة ذات بعد واحد (1D-DWT) مرتين على صفوف مكون الصورة، مـرة

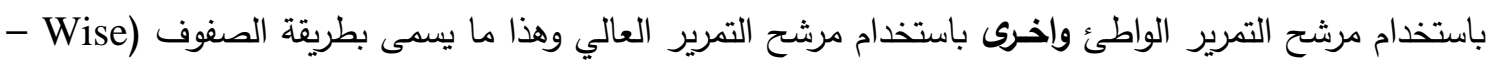

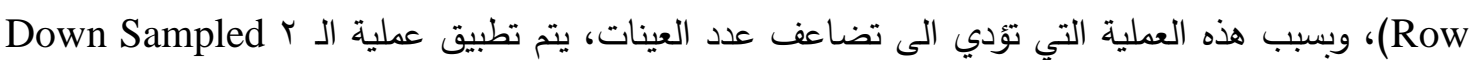

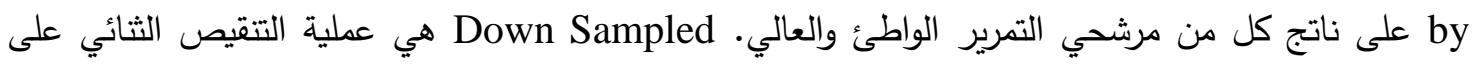

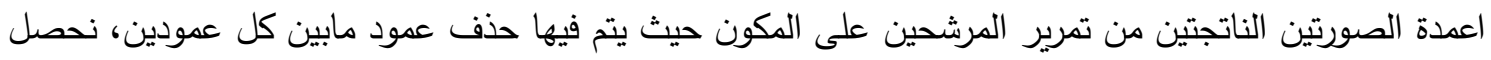

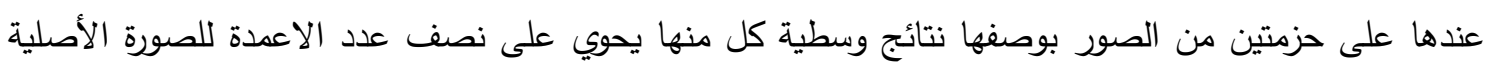
وهاتان الحزمتان تمثلان التقريب والتفاصيل لمكون الصورة. وفي الخطوة الثانية يطبق ايضاً تحويل المويجة ذات

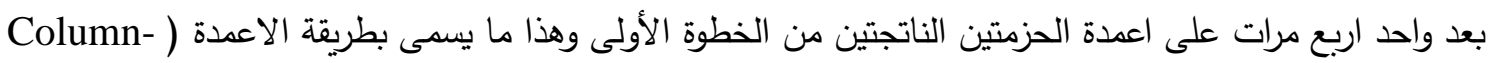

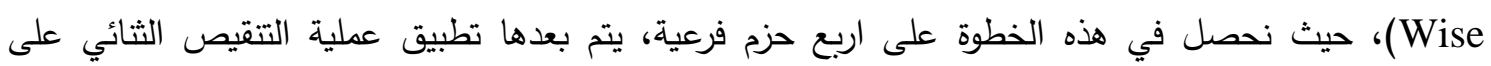

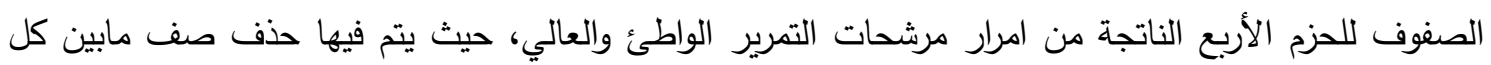

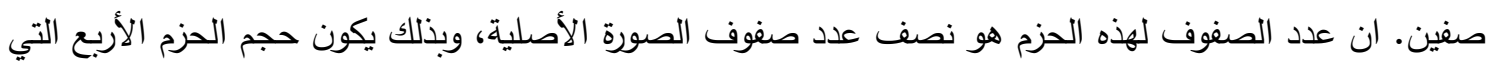

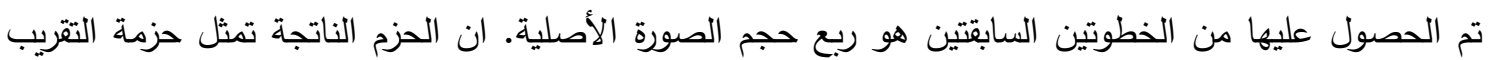
Details ( وهي تحوي المعلومات للصورة وتمثل بـ ALL (Approximation sub band) (sub bands وتمثل بـ Horizontal, Vertical, Diagonal ) HLH , VHL, DHH على التوالي) وهذه الحزم ليس فيها معلومات متكررة أي أن المعلومات الموجودة في HLH غير موجودة في كل من VHL , DHH وهذا ينطبق على جميع الحزم. اي انه يتم الحصول على اربع حزم فرعية في كل مرحلة تحليل، للاستمرار في التحليل الى المستوى الثاني يتم بأخذ الحزمة الفرعية ALL حيث يطبق عليها تحويل المويجة وبنفس الخطوات

تم استخدام مرشحات دبجي ثنائي التعامد 9/7 و 5/3 في تحويل المويجة، وهي مرشحات المويجة الافتراضية المستخدمة في معيار JPEG2000، أن مرشح التحليل 9/7 يتكون من 9 معاملات لمرشح التمرير

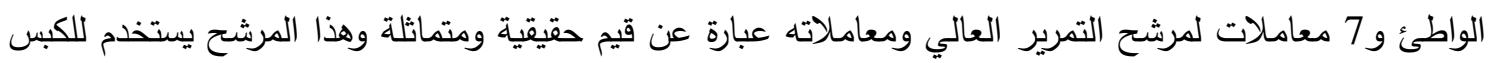
بفقدان، بينما مرشح التحليل 5/3 يستخدم للكبس دون فقدان وهو يتكون من 5 معاملات لمرشح التمرير الواطئ و 3

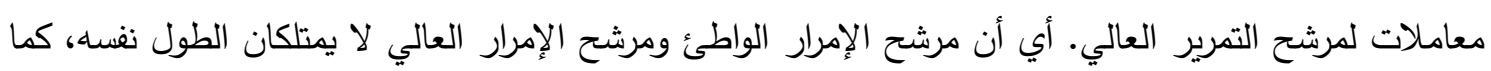

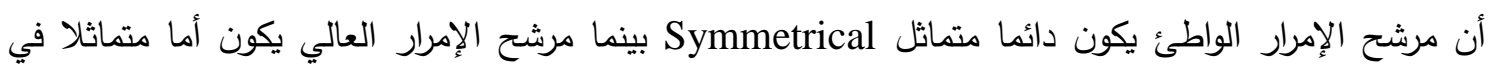

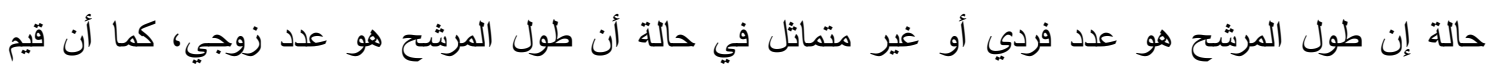

$$
\text { معاملات المرشح هي أما قيم حقيقية أو قيم صحيحة. }
$$

2.1.4 الخطوة الثانية لخوارزمية الكبس التكميم: هذه الخطوة مهمة لتساعد في تحقيق الكبس لأنها تساهم في فقدان المعلومات وتقليل دقة الحزم الفرعية. كل الحزم الناتجة من مرحلة التحويل المويجي يتم حساب قيمة التكميم لها عن طريق حساب قيمتي الدلتا (del) وحد العتبة(thr) ، حيث أن الدلتا تمثل الحجم على العرض للحزمة ماعدا 
الخلايا الصفرية، بينما قيمة حد العتبة تمثل العرض للخلايا الصفرية، أي أن كل حزمة يكون لها قيمة دلتا وقيمة حد عتبة خاصة. الثكل (4) يوضح خطوات تطبيق عملية التكميم على الحزم الفرعية.

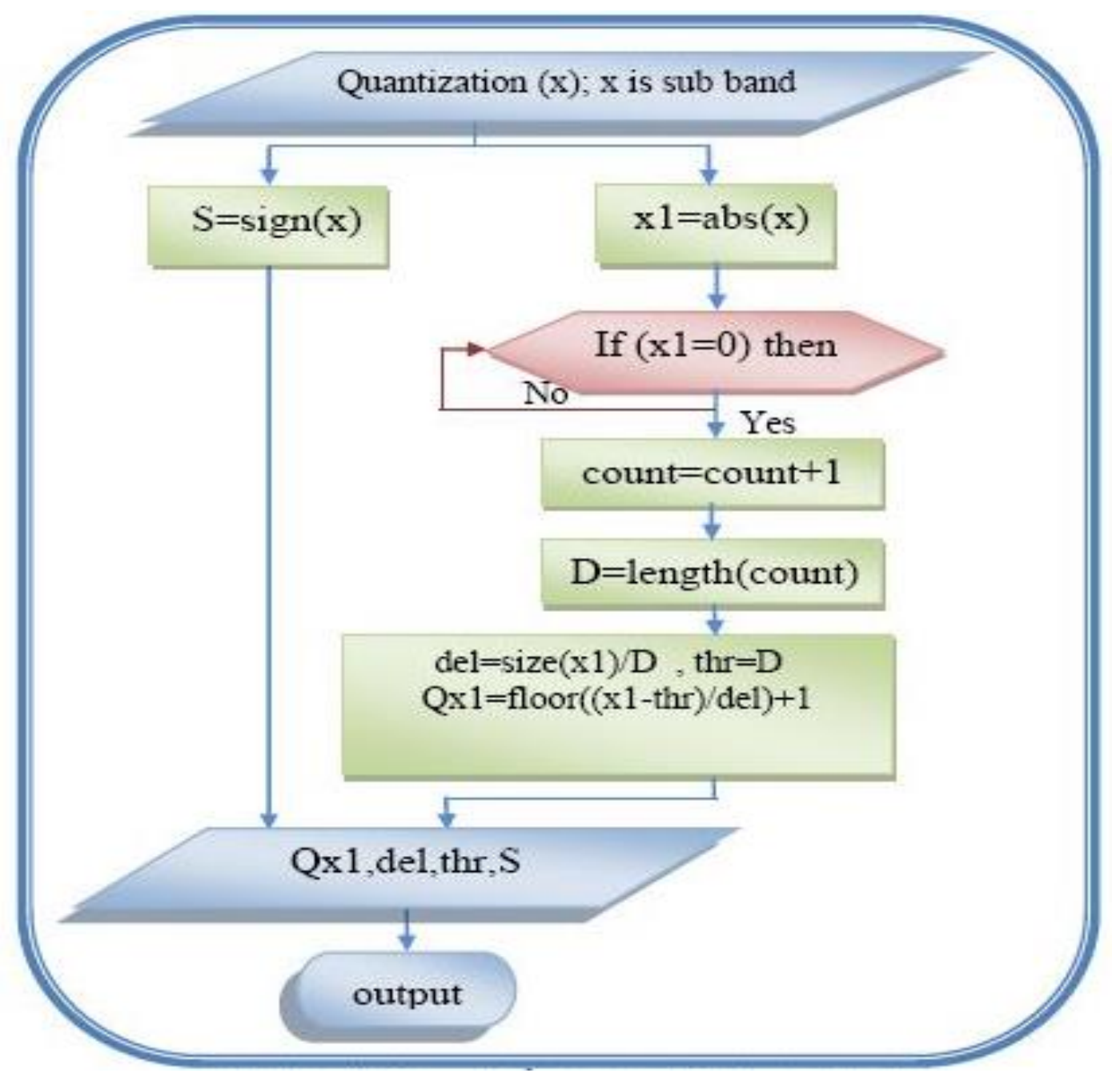

الثكل(4): المخطط الانسيابي لدالة التكميم (Quantization).

3.1.4 الخطوة الثالثة الترميز: وهي المرحلة الاخيرة في جهة المرمز ، حيث يتم ترميز معاملات المويجة الناتجة من عملية التكميم، الثكل (5) يبين خطوات تطبيق عملية الترميز الحسابي. ان الترميز الحسابي لا يمكنه ترميز مصفوفة ذات حجم كبير ، إذ ان اقصى حد يمكنه التعامل معه وترميزه بصورة صحيحة هو مصفوفة ذات حجم 24

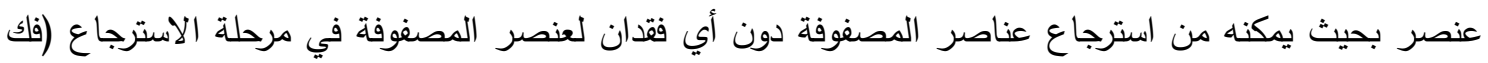
الترميز) . بما إننا نتعامل مع الصور ذات احجام كبيرة ومختلفة لذا تم تجزئة الصورة قبل تطبيق عملية الترميز

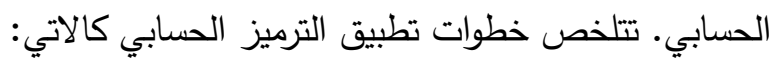
الخطوة الأولى هي تحويل المصفوفة Qxl ذات بعدين NxM الناتجة من عملية التكميم الى مصفوف ذات بعد واحد وهي idx بحجم [1,NxM]. وللحصول على نتائج ترميز كفؤة وبدون أية أخطاء وكذلك لتجاوز مشكلة التكرارات للعناصر ايضاً، يتم تجزئة المصفوفة idx الى كتل بحجم 8 عناصر ، وبذلك سيكون عدد الكتل الناتجة من التجزئة = NxM/8 ، وتمثل Blocks في المخطط عدد الكتل. الخطوة الثانية هي الترميز ، إذ أن دالة 
الترميز الحسابي ترمز كل كتلة بصورة مستقلة، للبدء بعملية الترميز يتم اولا ايجاد عناصر الكتلة وبدون تكرار

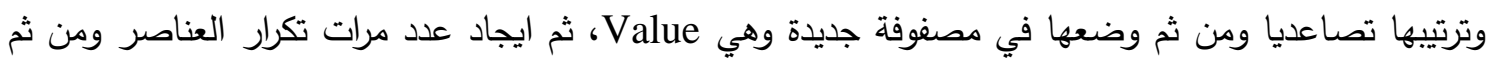
حساب الاحتمالية لهذه العناصر والاحتفاظ بها في مصفوفة prob، يتم بعد ذلك حساب المدى لكل عنصر في

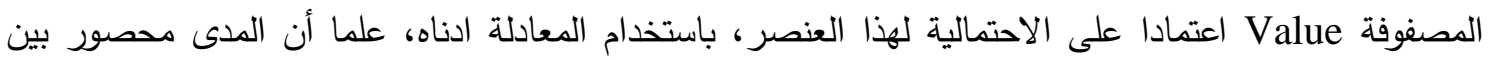
: $[0.0,0.1)$

High_range $(\operatorname{value}(j))=[$ High_range $\operatorname{sum}($ pro $)]$

low_range $(\operatorname{value}(\mathbf{j}))=\left[\begin{array}{ll}0 & \text { High_range }-1\end{array}\right]$

اي ان low _ range و مثل المدى الواطي و العالي للعنصر j في المصفوفة .Value 


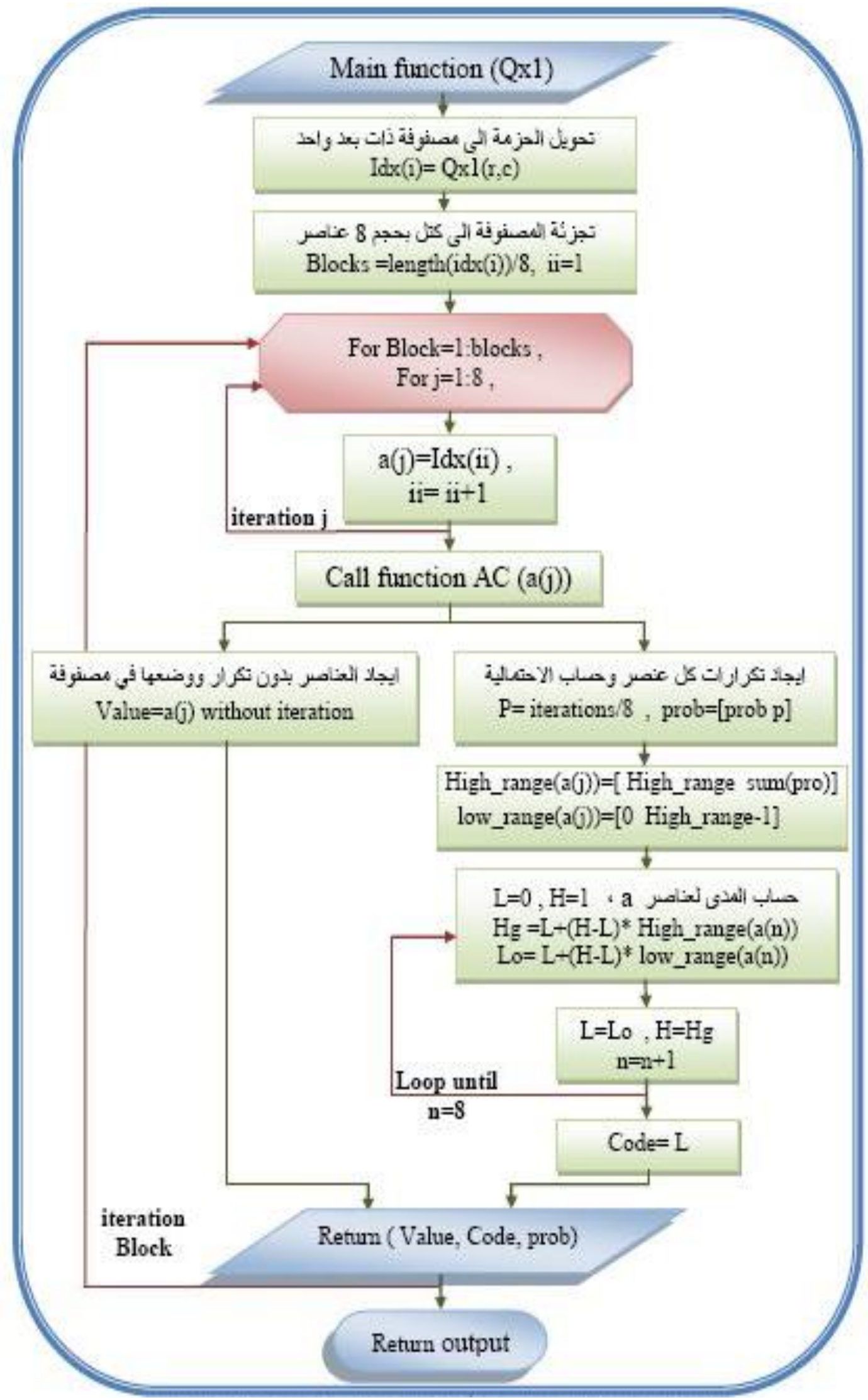

الثكل(5): المخطط الانسيابي لدالة الترميز الحسابي (Arithmetic coding). 
بعد حساب المدى لعناصر المصفوفة يتم عندها معالجة عناصر الكتلة a وإيجاد المدى لهم باستخدام

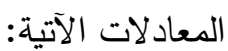

\section{$\mathbf{L}=\mathbf{0}, \mathbf{H}=\mathbf{1}$}

$\mathrm{Hg}=\mathrm{L}+(\mathrm{H}-\mathrm{L}) *$ High range $(\mathbf{a}(\mathrm{n}))$

Lo $=\mathbf{L}+(\mathbf{H}-\mathbf{L}) *$ low_range $(\mathbf{a}(\mathbf{n}))$

\section{$\mathrm{L}=\mathrm{Lo}, \mathrm{HEHg}$}

بعد معالجة كل عناصر الكتلة، فان اخر عنصر فيها المدى الواطئ له يكون هو الترميز لهذه الكتلة (اي Code = L

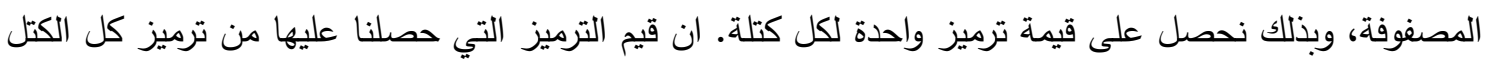
يتم الاحتفاظ بها في مصفوفة واحدة ومن ثم تحويلها الى صيغة نصية وخزنها او ارسالها.

2.4 في جهة فك الترميز Decoder Side:

1.2 .4 الخطوة الأولى لفك الترميز: إن اول خطوة لفك الترميز بعد استلام الصيغة النصية هي تحويلها إلى صيغتها الأصلية، وهي المصفوفة التي تحتوي على قيم الترميز لكتل الحزمة. إن جهة الترميز وفك الرمز لديهما

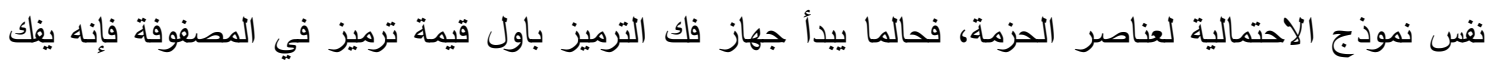

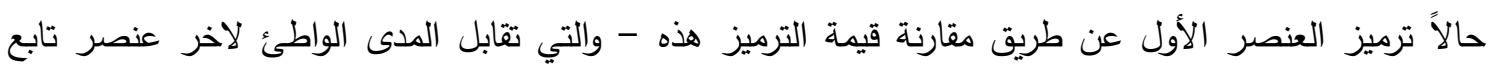

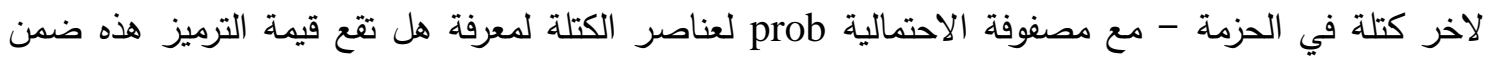

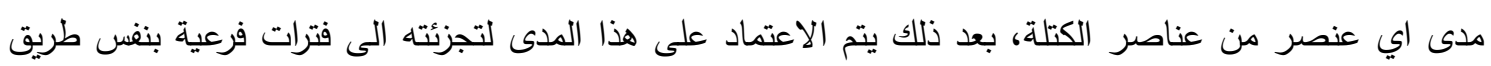
الترميز المذكورة في جهة المرمز مع عملية مقارنة مع هذه الفترات الفرعية في كل عملية تجزئة لفك ترميز

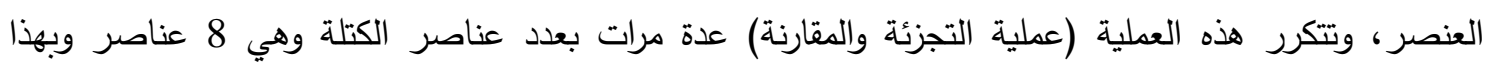

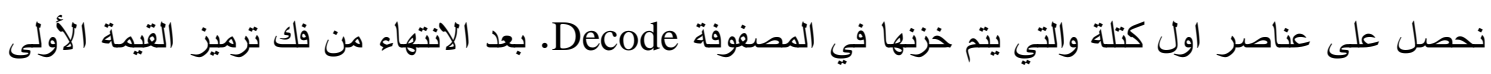
المصفوفة الترميز Code يتم عندها اكمال فك ترميز باقي قيم الترميز للمصفوفة Code وبنفس الطريقة السابقة، وبذلك نتمكن من استرجاع كل عناصر الحزمة وبدون أي فقدان. الثكل (6) يبين خطوات تطبيق فلك الترميز

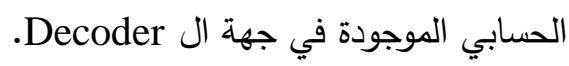




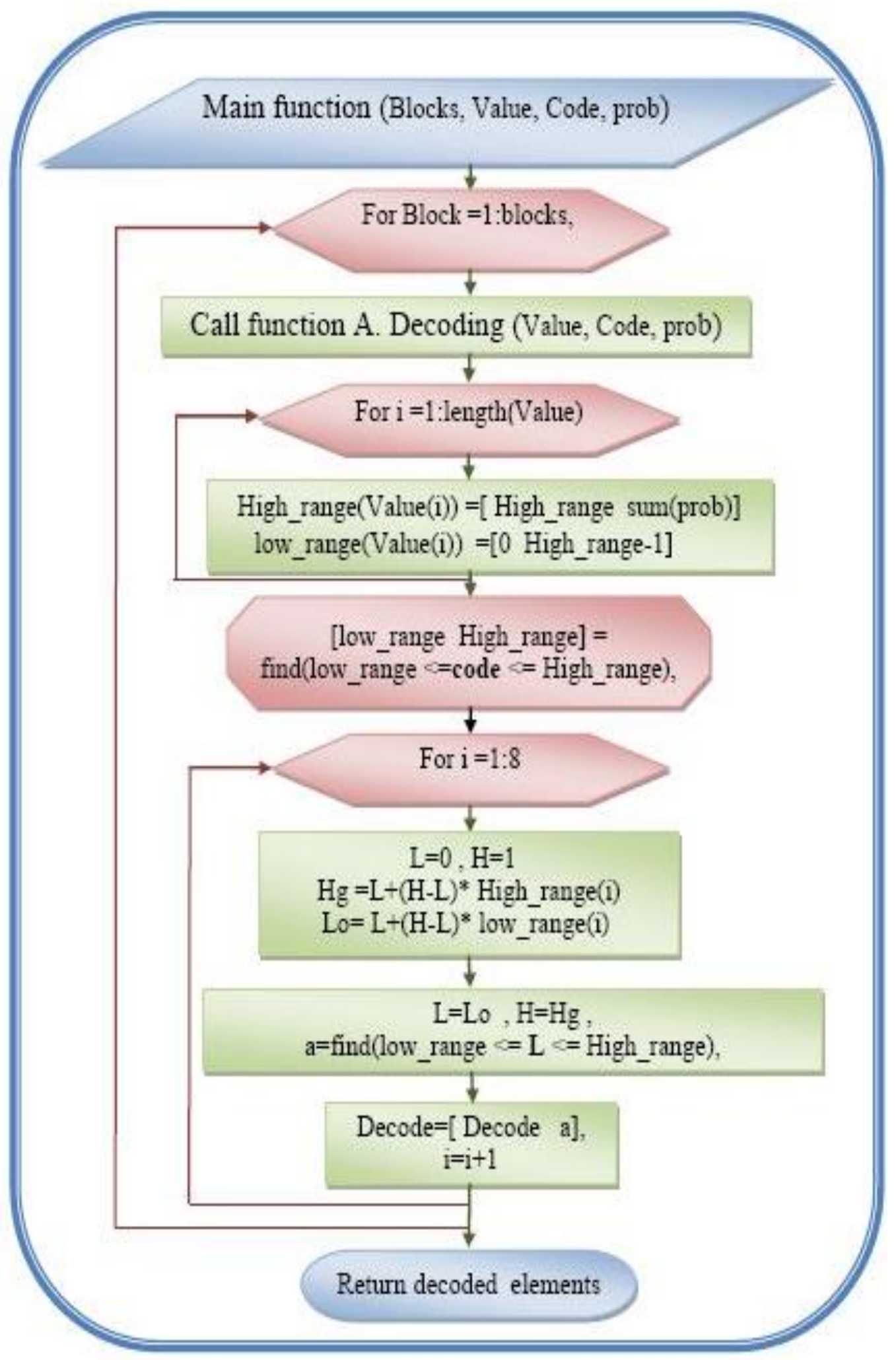

الشكل(6): المخطط الانسيابي لدالة فك الترميز (Arithmetic decoding). 
2.2.4 الخطوة الثانية لفك الترميز معكوس التكميم: بعد الانتهاء من فلك ترميز الحزمة يتم تطبيق دالة معكوس

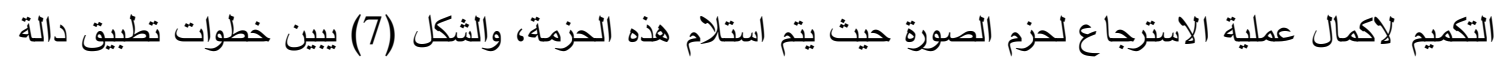
معكوس التكميم. لتطبيق عملية معكوس التكميم تضرب كل حزمة بقيمة الالتا الخاصة بها اولا ثم تُقسم قيمة الدلتا هذه على 2 ومن ثم يطرح الناتج من قيمة حد العتبة ثانية بعدها يتم جمع ناتج اولا وثانية لاسترجاع الحزمة.

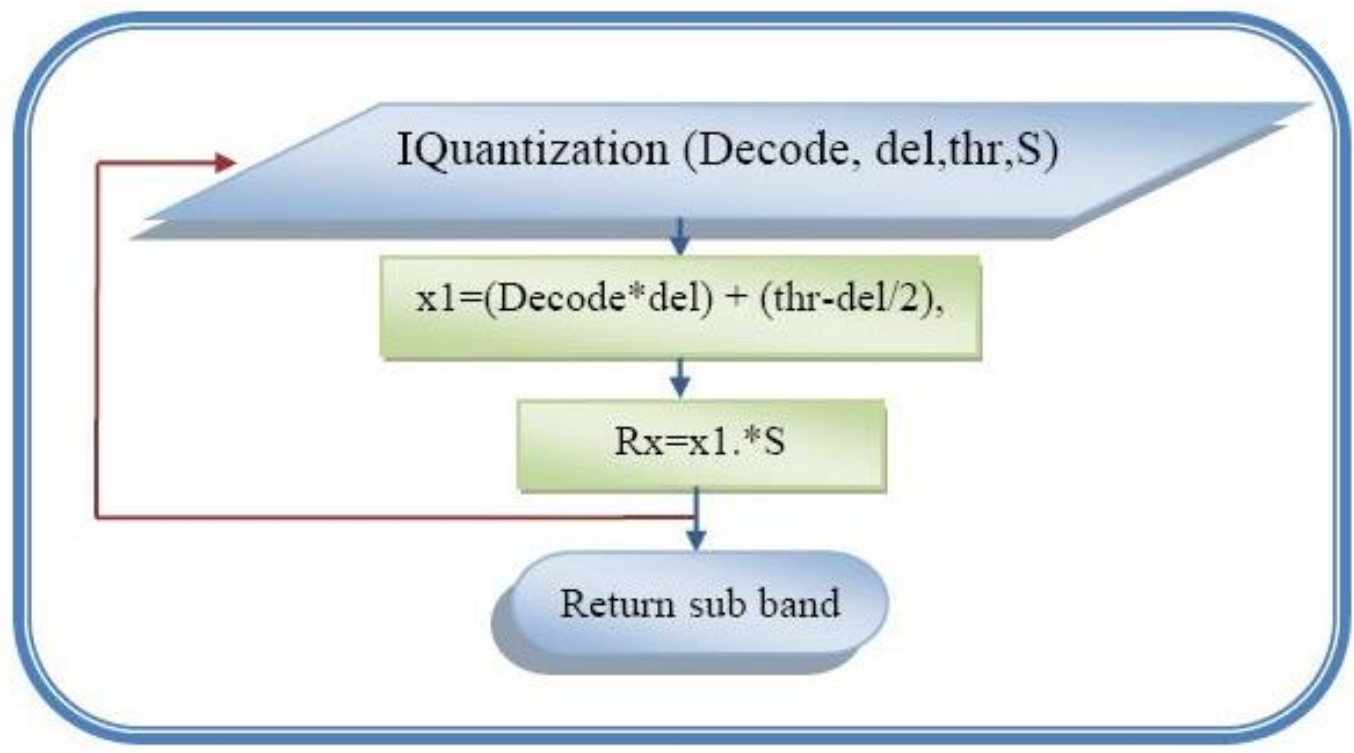

\section{الثكل(7): المخطط الانسيابي لعملية عكس التكميم.}

3.2 .4 الخطوة الثالثة معكوس تحويل المويجة: تطبيق معكوس تحويل المويجة (2D- IDWT) ذات بعدين

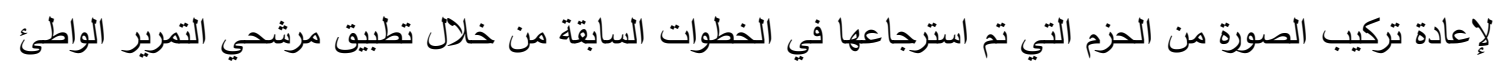

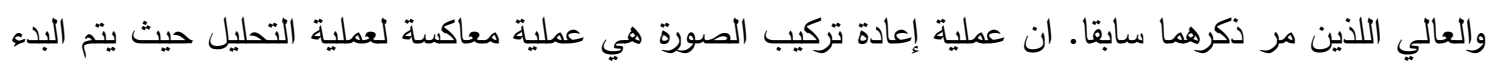

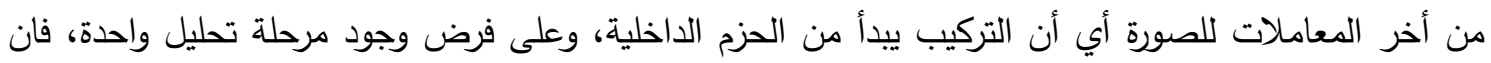
معكوس تحويل المويجة ذات بعدين ينجز بخطوتين: في الخطوة الاولى اجراء عملية الـ Up sampled by 2 على اعمدة كل حزمة من الحزم الاربعة الناتجة من عملية عكس التكميم (ALL, HLH, VHL, DHH) وذلك عن طريق ادراج عمود قيمته اصفار بين كل عموديين متتالين، وبعد ذلك يتم تطبيق تحويل المويجة (IDWT) وكما يأتي: i.. تطبيق تحويل المويجة المعكوس على صفوف الحزمة ALL باستخدام مرشح التمرير الواطئ بطريقة الالتفاف الرياضي، وتطبيقه ايضا على صفوف الحزمة HLH باستخدام مرشح التمرير العالي ثم يتم جمع ناتج

$$
\text { الحزمتين للحصول على AL. }
$$

ii تطبيق معكوس تحويل المويجة على صفوف الحزمة VHL باستخدام مرشح التمرير الواطئ بطريقة الالتفاف الرياضي، وتطبيقه ايضا على صفوف الحزمة DHH باستخدام مرشح التمرير العالي ثم يتم جمع ناتج الحزمتين للحصول على AH. وفي الخطوة الثانية اجراء عملية الـ Up sampled by مرة اخرى ولكن على صفوف الحزمتين الناتجتين من الخطوة السابقة ( AL ، AH ) وذلك عن طريق ادراج صف قيمته اصفار بين كل صفين متتالين، وبعد ذلك يتم 
تطبيق معكوس تحويل المويجة على أعمدة الحزمة AL باستخدام مرشح التمرير الواطئ بطريقة الالتفاف الرياضي، وتطبيقه ايضا على اعمدة الحزمة AH باستخدام مرشح التمرير العالي ثم يتم جمع ناتج الحزمتين للحصول على الصورة او احدى المكونات اللونية للصورة الملونة.

بعد اكمال عملية اعادة تركيب كل المكونات اللونية للصورة يتم عندها تطبيق المعادلات الآتية لإعادة هذه المكونات الى الفضاء RGB:

$\mathbf{G}=\operatorname{round}((\mathbf{Y}-(\mathrm{U}+\mathrm{V}) / 4))$

$\mathbf{R}=\mathbf{U}+\mathbf{G}$

$\mathbf{B}=\mathbf{V}+\mathbf{G}$

وفيما يأتي نتائج تطبيق الخوارزمية المقترحة لمعيار JPEG2000 باستخدام مرشحات دبجي ثنائي التعامد 9/7 و 5/3 في تحويل المويجة:

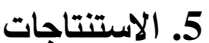

ان الثكل (8) يبين نتائج تطبيق خوارزمية الكبس المقترحة باستخدام المشحين على الصور الطبيعية Girl الرمادية والملونة. ان نتائج مقياس PSNR و MSE ووقت التتفيذ التي تم الحصول عليها من تطبيق باست

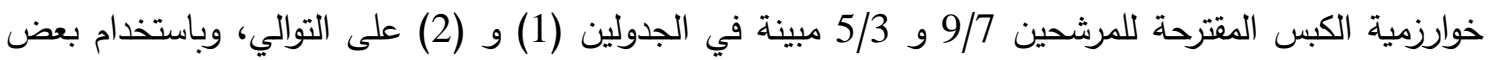

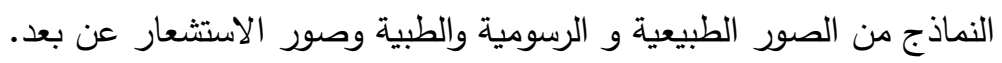




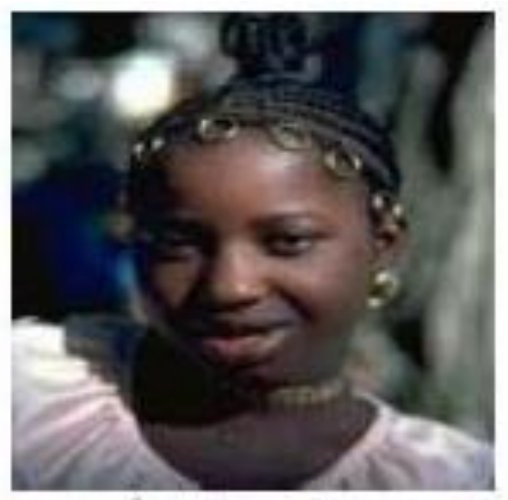

الصورة الملوئة

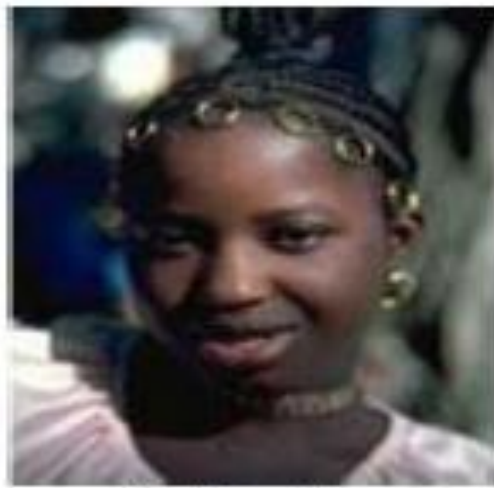

الصورة المئونة
الأصلية

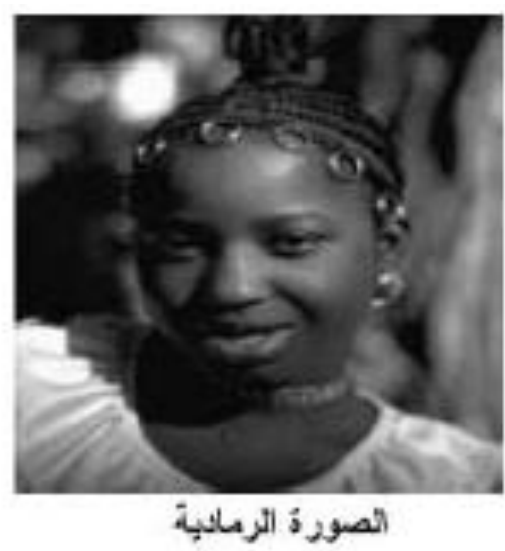

(a)
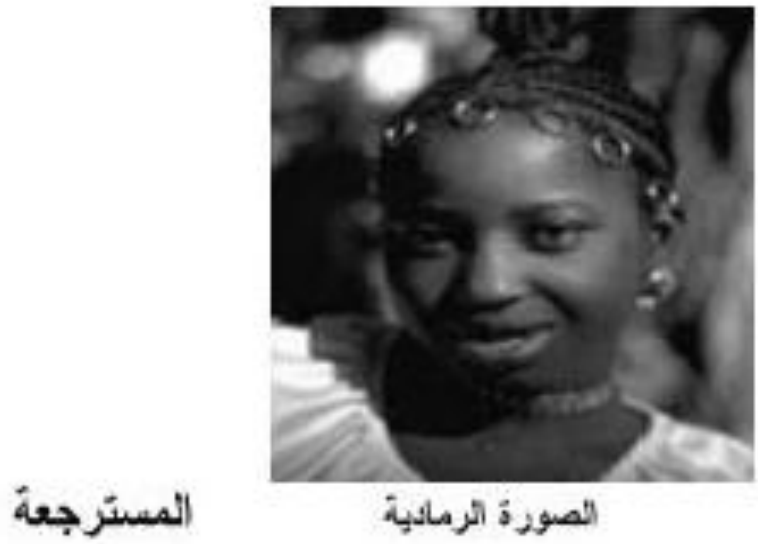

باستخدام المرشيح 9/7

(b)

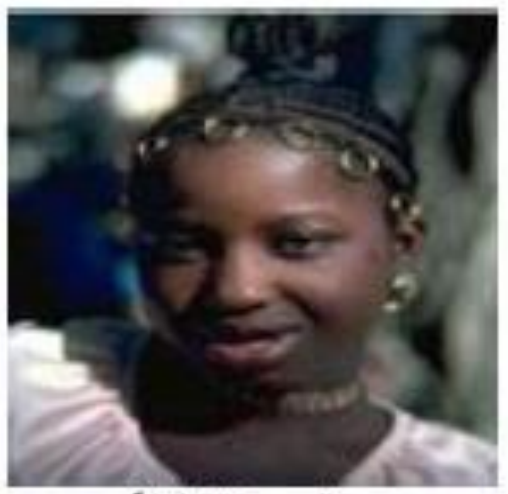

الصورة: المئونة

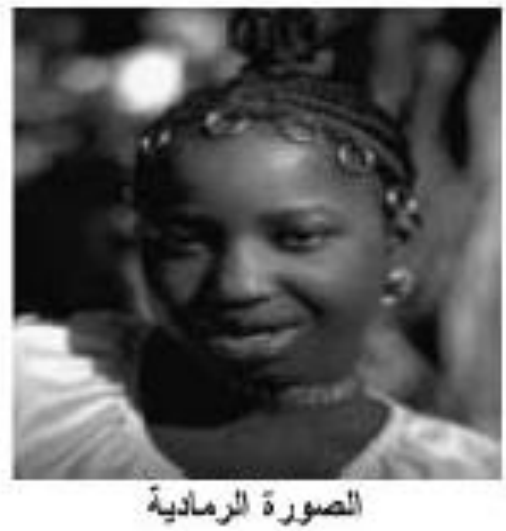

باستخدام المرشي 5/3

الثكل(8): تطبيق خوارزمية الكبس المقترحة على صورة Girl، (a) الصورة الاصلية، (b) الصورة المسترجعة باستخدام المرشح 9/7، (c) الصورة المسترجعة باستخدام المرشح 5/3. 
أظهرت النتائج المثبتة في الجدولين أن قيم مقياس PSNR للصور الملونة أعلى منها عند الصور الرمادية لجميع نماذج الصور المعتمدة في البحث، ووقت التتفيذ المستغرق في الصور الملونة أعلى منها في الصور الرمادية ولجميع النماذج ايضا وهذا ينطبق على المرشحين، والثكلين (9) و (10) يبينان نتائج المقارنة بين الصور الملونة والرمادية التي تم الحصول عليها عند اعتماد المرشحين 9/7 و 5/3 على التوالي. ان الخوارزمية المقترحة أكثر كفاءة عندما تتعامل مع الصور ذات الأحجام الكبير وذات لون عمقي كبير

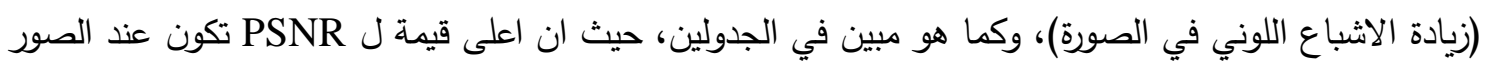

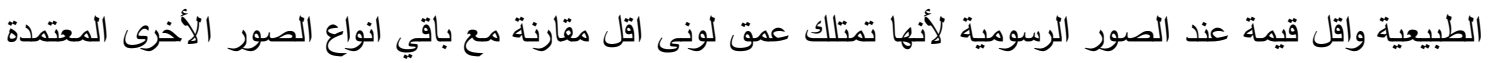
في البحث. الجدول (1): النتائج التجريبية لتطبيق الخوارزمية المقترحة باستخدام مرشح ديجي 9/7 لانواع من الصور.

\begin{tabular}{|c|c|c|c|c|c|c|c|c|}
\hline \multicolumn{9}{|c|}{ Daubechies $9 / 7$ biorthogonal filter } \\
\hline \multicolumn{3}{|c|}{ Gray Image } & \multicolumn{3}{|c|}{ Color Image } & \multirow[b]{2}{*}{ Size } & \multirow[b]{2}{*}{ Images } & \multirow[b]{2}{*}{ Types } \\
\hline $\begin{array}{l}\text { Time } \\
\text { (sec.) }\end{array}$ & MSE & $\begin{array}{l}\text { PSNR } \\
\text { (dB) }\end{array}$ & $\begin{array}{l}\text { Time } \\
\text { (sec.) }\end{array}$ & MSE & $\begin{array}{c}\text { PSNR } \\
\text { (dB) }\end{array}$ & & & \\
\hline 82.67 & 925.31 & 18.4679 & 225.51 & 3.1931 & 53.519 & $512 * 512$ & Baby & \multirow{5}{*}{ 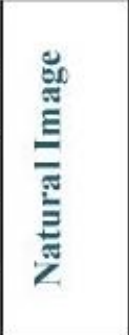 } \\
\hline 8.203 & 2.2474 & 14.6141 & 15.219 & 6.8994 & 43.4412 & $256^{*} 256$ & Koala & \\
\hline 7.453 & 1.4480 & 16.5233 & 14.953 & 4.3704 & 49.3943 & $256^{*} 256$ & Girl & \\
\hline 6.703 & 3.0464 & 13.2929 & 18.563 & 9.7719 & 38.9829 & $256^{*} 256$ & Fruit & \\
\hline 7.625 & 1.3466 & 16.8385 & 19.875 & 4.7291 & 48.3809 & $256^{*} 256$ & Bike & \\
\hline 6.890 & 3.3280 & 12.9089 & 21.328 & 1.0305 & 38.2193 & $256^{*} 256$ & Duck & \multirow{3}{*}{ 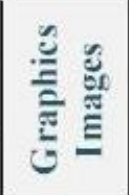 } \\
\hline 7.766 & 4.9894 & 11.1504 & 21.531 & 1.6098 & 32.4173 & $256^{*} 256$ & Child & \\
\hline 1.844 & 2.7875 & 13.6786 & 4.0790 & 9.1710 & 39.7584 & $128 * 128$ & Bunny & \\
\hline 6.829 & 2.2954 & 14.5223 & 18.047 & 7.2508 & 43.1258 & $256 * 256$ & $\begin{array}{c}\text { Medical } \\
1\end{array}$ & \multirow{2}{*}{ 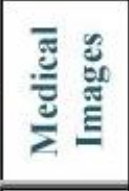 } \\
\hline 7.172 & 1.7370 & 15.7329 & 20.062 & 4.8457 & 49.4272 & $256^{*} 256$ & $\begin{array}{c}\text { Medical } \\
2\end{array}$ & \\
\hline 7.703 & 1.6339 & 15.9985 & 22.687 & 5.2463 & 47.0593 & $256 * 256$ & Image1 & \multirow{2}{*}{ 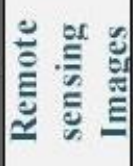 } \\
\hline 7.406 & 1.1679 & 17.4567 & 19.281 & 4.3393 & 49.5184 & $256 * 256$ & Image 2 & \\
\hline
\end{tabular}


الجدول (2): النتائج التجريبية لتطبيق الخوارزمية المقترحة باستخدام مرشح ديجي 5/3 لانواع من الصور.

\begin{tabular}{|c|c|c|c|c|c|c|c|c|}
\hline \multicolumn{9}{|c|}{ Daubechies $5 / 3$ biorthogonal filter } \\
\hline \multicolumn{3}{|c|}{ Gray Image } & \multicolumn{3}{|c|}{ Color Image } & \multirow[b]{2}{*}{ Size } & \multirow[b]{2}{*}{ Images } & \multirow[b]{2}{*}{ Types } \\
\hline $\begin{array}{l}\text { Time } \\
\text { (sec.) }\end{array}$ & MSE & $\begin{array}{c}\text { PSNR } \\
\text { (dB) }\end{array}$ & $\begin{array}{l}\text { Time } \\
\text { (sec.) }\end{array}$ & MSE & $\begin{array}{c}\text { PSNR } \\
\text { (dB) }\end{array}$ & & & \\
\hline $\begin{array}{c}94.34 \\
4 \\
\end{array}$ & 810.651 & 19.0425 & 280 & 2.570 & 56.3555 & $512 * 512$ & Baby & \multirow{5}{*}{ 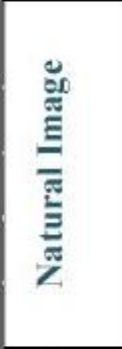 } \\
\hline 8.141 & 1.5899 & 16.1171 & 20.797 & 4.1258 & 50.1389 & $256 * 256$ & Koala & \\
\hline 8.016 & 809.8615 & 19.0467 & 21.266 & 2.4813 & 56.7712 & $256^{*} 256$ & Girl & \\
\hline 7.812 & 2.4518 & 14.2360 & 22.438 & 6.3849 & 44.5110 & $256^{*} 256$ & Fruit & \\
\hline 7.187 & 1.6059 & 16.0737 & 20.2040 & 2.7202 & 55.6082 & $256 * 256$ & Bike & \\
\hline 8.453 & 4.2063 & 11.8918 & 21.437 & 8.7569 & 40.3413 & $256 * 256$ & Duck & \multirow{3}{*}{ 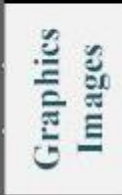 } \\
\hline 7.532 & 4.9504 & 11.1844 & 21.563 & 1.0363 & 38.1417 & $256 * 256$ & Child & \\
\hline 7.579 & 2.4186 & 14.2952 & 20.750 & 4.7912 & 48.2156 & $256 * 256$ & Bunny & \\
\hline 7.125 & 1.6897 & 15.8527 & 20.953 & 6.1031 & 45.2053 & $256 * 256$ & $\begin{array}{c}\text { Medical } \\
1 \\
\end{array}$ & \multirow{2}{*}{ 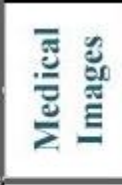 } \\
\hline 7.141 & 1.3010 & 16.9880 & 20.093 & 3.2768 & 54.4125 & $256^{*} 256$ & $\begin{array}{c}\text { Medical } \\
2\end{array}$ & \\
\hline 8.047 & 1.2785 & 17.0637 & 21.047 & 3.9402 & 50.7805 & $256 * 256$ & Image1 & \multirow{2}{*}{ 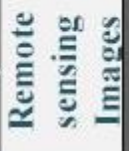 } \\
\hline 7.687 & 1.3611 & 16.7918 & 20.188 & 3.360 & 52.6192 & $256 * 256$ & Image2 & \\
\hline
\end{tabular}

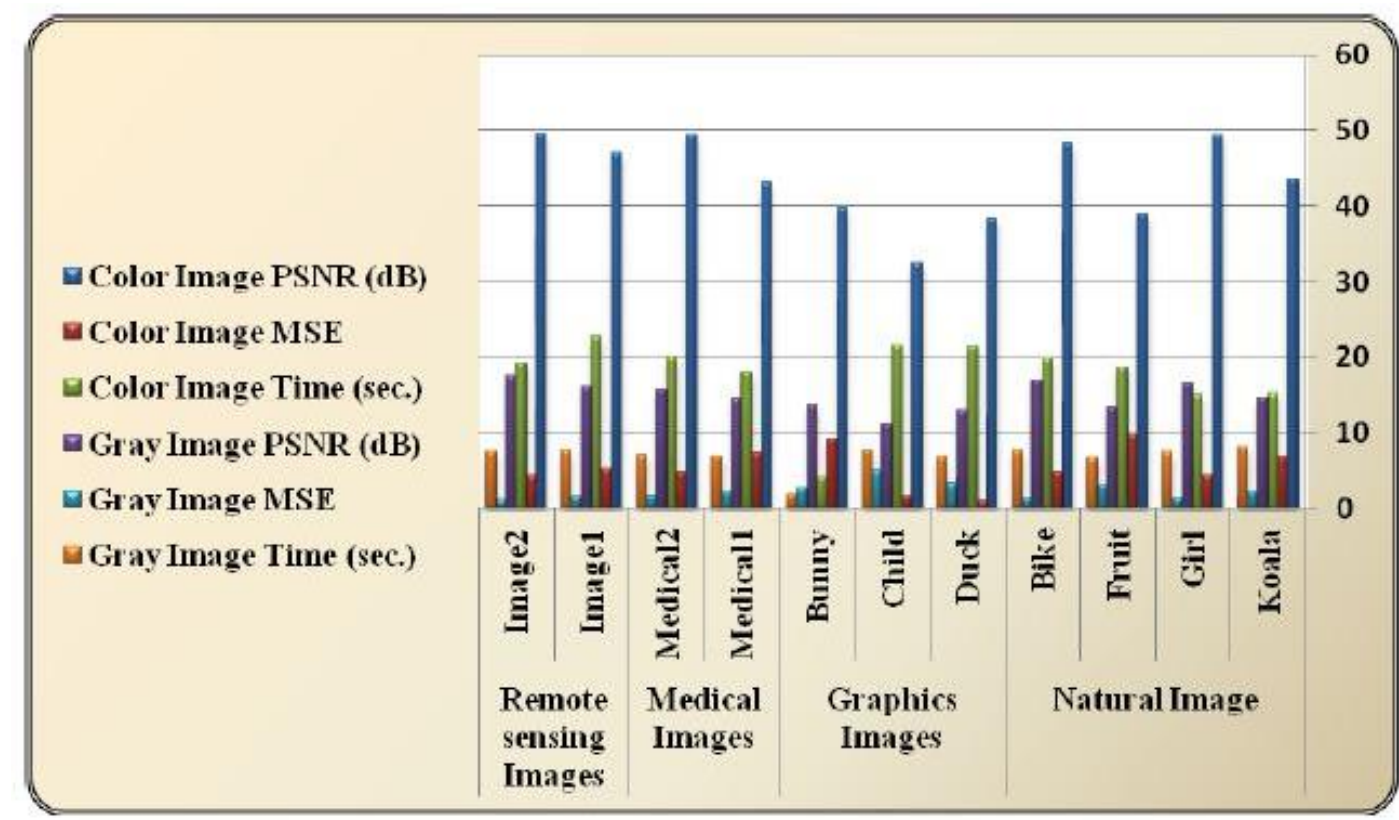

الشكل(9): مخطط مقارنة تطبيق خوارزمية الكبس المقترحة لمعيار JPEG2000 لمرشح 9/7 بين الصور

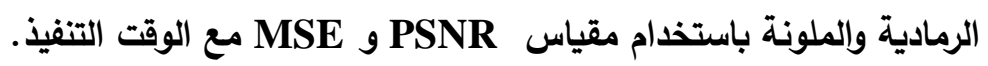




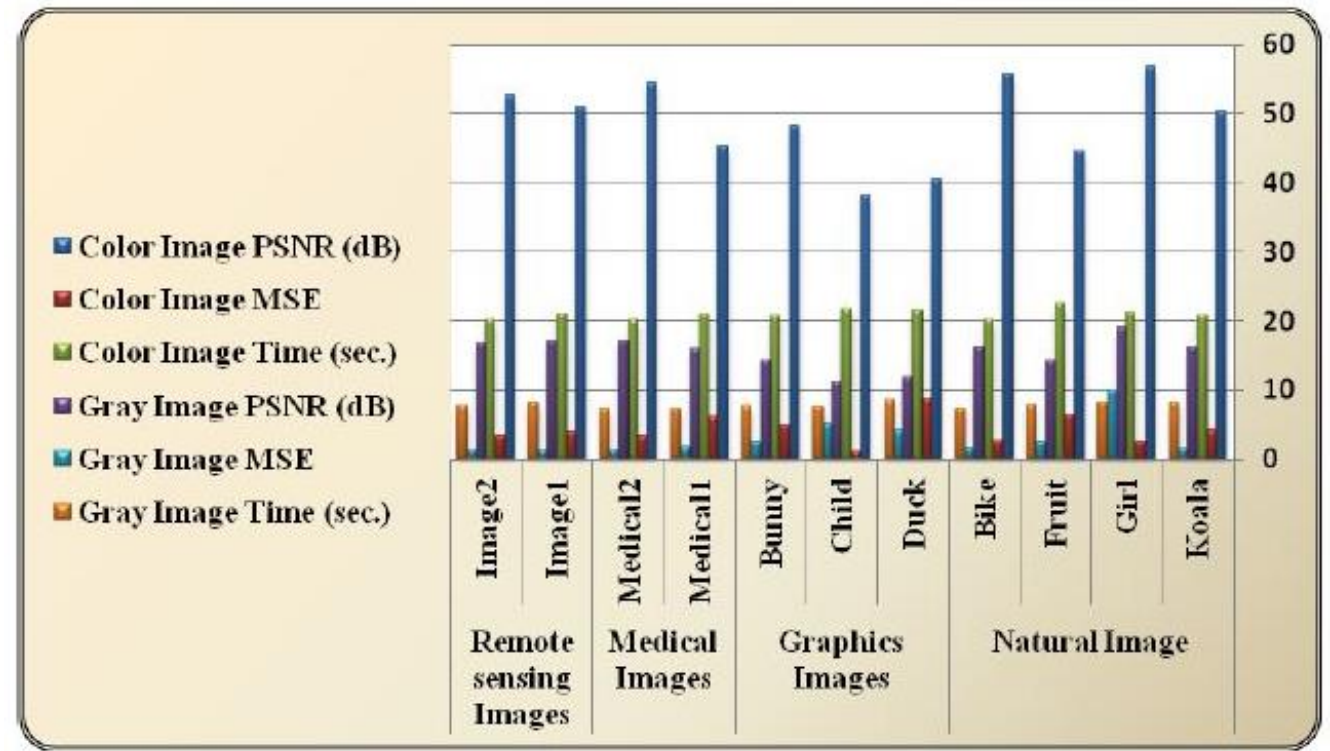

الثكل(10): مخطط مقارنة تطبيق خوارزمية الكبس المقترحة لمعيار JPEG2000 لمرشح 5/3 بين الصور

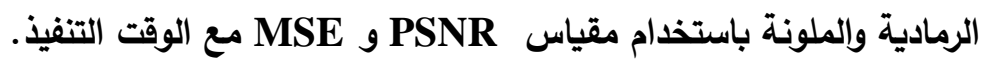

تم ايضاً اجراء مقارنة لنتائج خوارزمية الكبس المقترحة بين المرشحين 9/7 و 5/3 للصور الملونة والرمادية والثكل (11) يبين نتائج المقارنة بين المرشحين بالنسبة إلى الصور الملونة بينما الثكل (12) يبين نتائج المقارنة

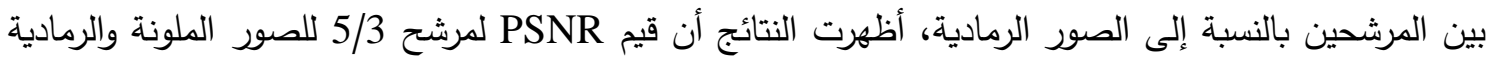
اعلى مقارنة مع المرشح 9/7 لكنه يستغرق وقت اطول.

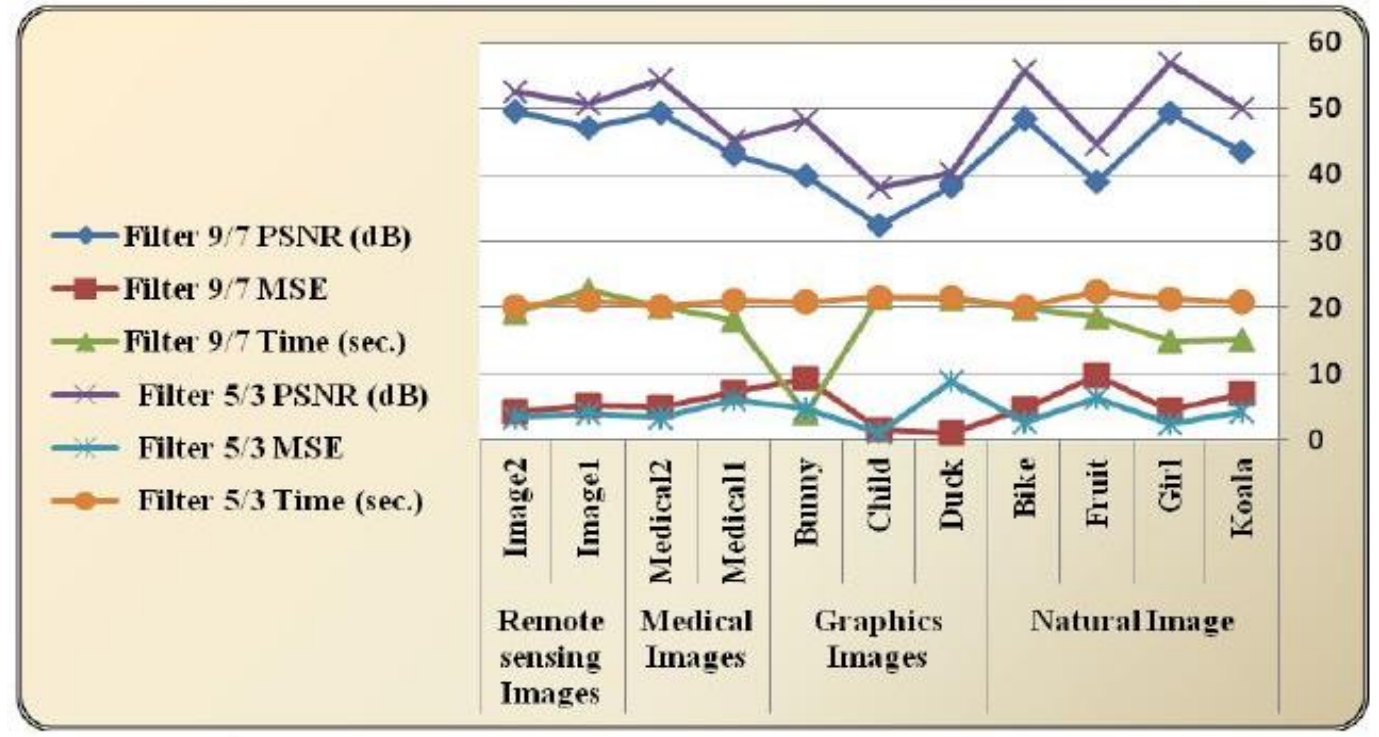

الثكل(11): مخطط مقارنة تطبيق الخوارزمية المقترحة باستخدام مرشح 9/7 و 5/3 لأنواع من الصور الملونة. 
$\multimap$ Filter 9/7 PSNR (dB)

- Filter $9 / 7$ MSE

$\longrightarrow$ Filter 9/7 Time (sec.)

Filter $5 / 3$ PSNR (dB)

$\longrightarrow$ Filter 5/3 MSE

$\multimap$ Filter $5 / 3$ Time (sec.)

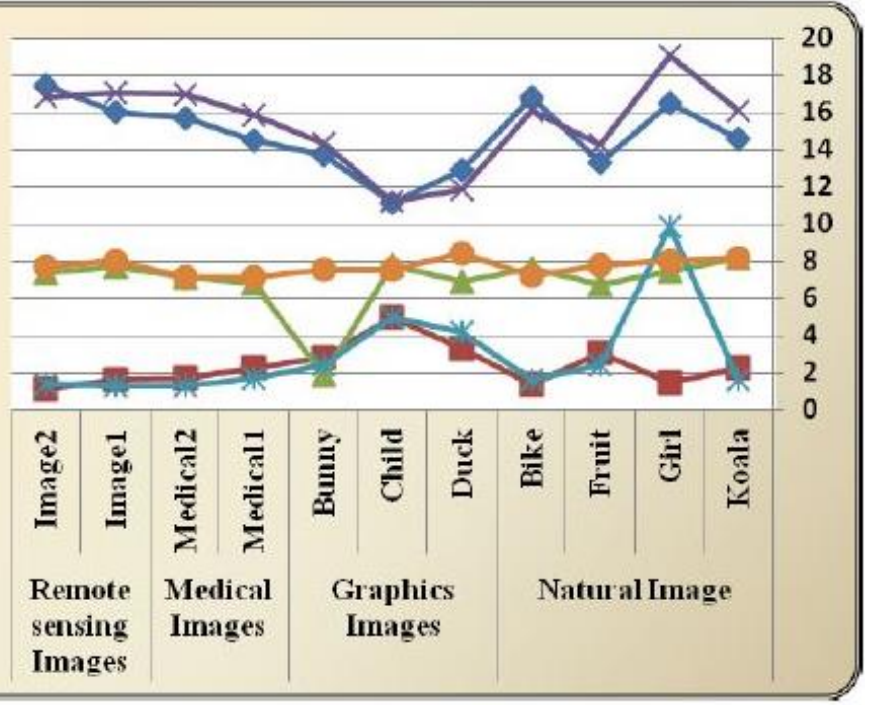

الثكل(12): مخطط مقارنة تطبيق الخوارزمية المقترحة باستخدام مرشح 9/7 و 5/3 لأنواع من الصور الرمادية.

تم ايضا تطبيق الخوارزمية المقترحة لمعيار JPEG2000 باستخدام المرشحين على نماذج من الصور الملونة المستخدمة في خوارزميتي SPECK-Color و واجراء مقارنة بين نتائج التطبيق التي حصلنا عليها مع نتائج الخوارزميتين باعتماد مقياس PSNR. ان نتائج قيم PSNR التي تم الحصول عليها من

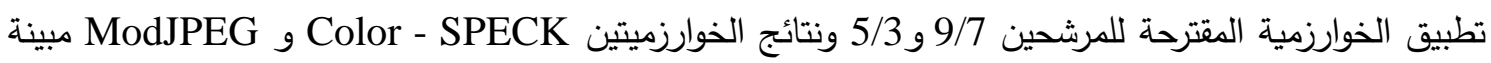
في الجدولين (3) و (4) على التوالي. اظهرت النتائج أن قيم PSNR لخوارزمية الكبس المقترحة لمعيار

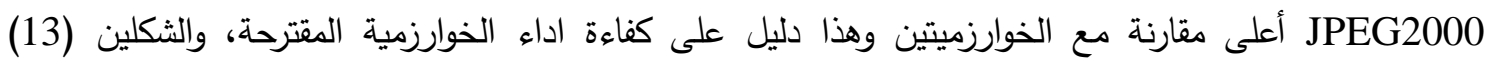

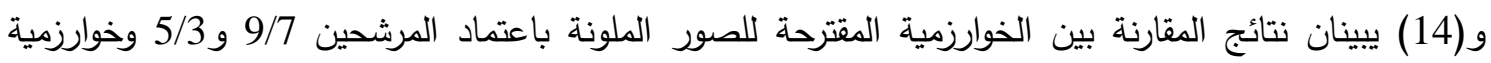
SPECK Color و و ModJPEG على التوالي. الجدول(3): نتائج التطبيق العملي للخوارزمية المقترحة لـ JPEG2000 باستخدام المرشحين ونتائج خوارزمية .Color-SPECK

\begin{tabular}{|c|c|c|c|c|}
\hline $\begin{array}{c}\text { Color-SPECK } \\
\text { PSNR }\end{array}$ & PSNR $\backslash \mathbf{5} / \mathbf{3}$ & PSNR $\backslash \mathbf{9 / 7}$ & Size & Images \\
\hline $38.45 \mathrm{~dB}$ & 59.0030 & $55.0255 \mathrm{~dB}$ & $512 * 512 * 3$ & Lena \\
\hline $34.98 \mathrm{~dB}$ & 56.0376 & $49.4270 \mathrm{~dB}$ & $512 * 512 * 3$ & Barbara \\
\hline $36.71 \mathrm{~dB}$ & 62.6582 & $58.7442 \mathrm{~dB}$ & $512 * 512 * 3$ & Goldhill \\
\hline
\end{tabular}

الجدول(4): نتائج التطبيق العملي للخوارزمية المقترحة لـ JPEG2000 باستخدام المرشحين ونتائج خوارزمية ModJPEG

\begin{tabular}{|c||c|c|c||c|}
\hline ModJPEG \PSNR & PSNR $\backslash 5 / 3$ & PSNR $\backslash 9 / 7$ & Size & Images \\
\hline \hline 32.027 & 56.4851 & 51.9197 & $256 * 256 * 3$ & House \\
\hline 29.6878 & 44.6991 & 43.4497 & $256 * 256 * 3$ & Boat \\
\hline 26.477 & 47.7402 & 42.6719 & $256 * 256 * 3$ & Sailboat \\
\hline
\end{tabular}




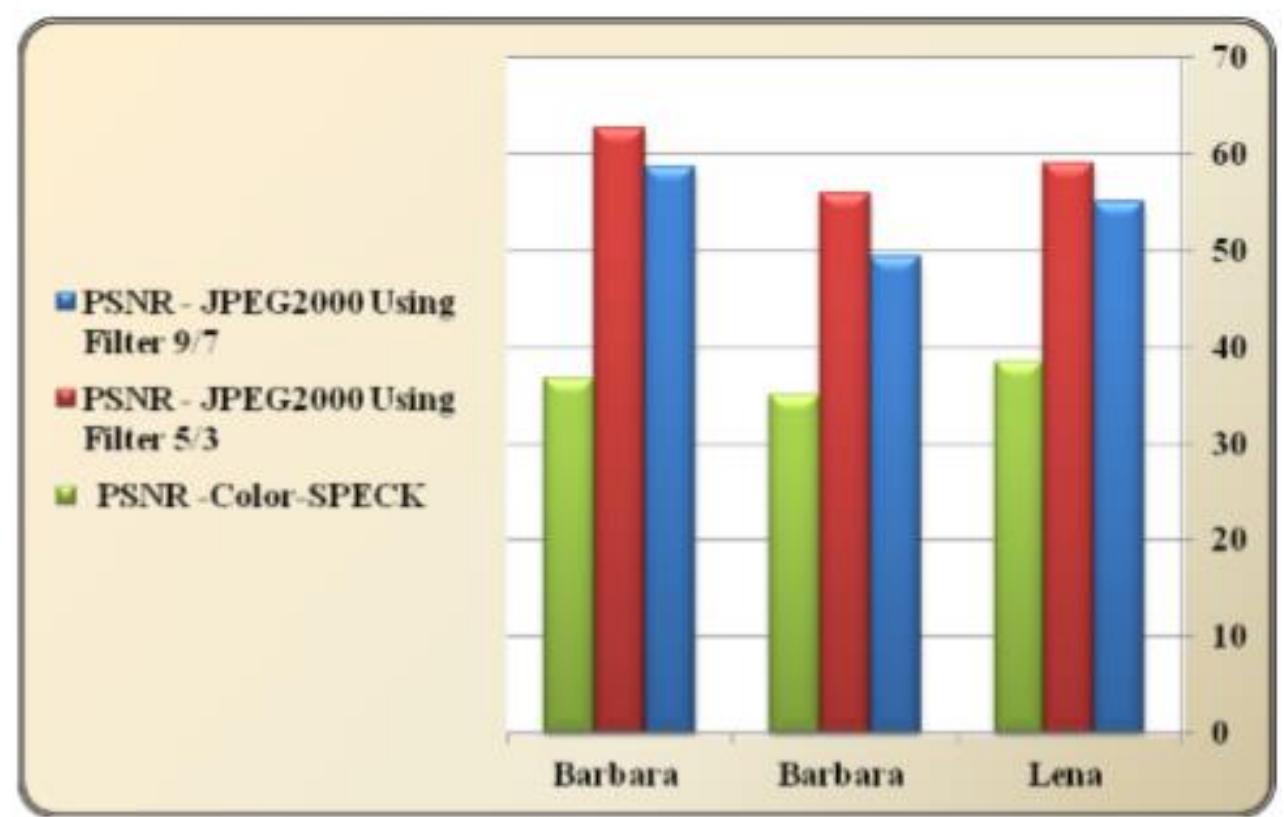

الثكل(13): مخطط مقارنة تطبيق الخوارزمية المقترحة لـ JPEG2000 باستخدام المرشحين مع خوارزمية PSNR للصور الملونة باستخدام المقياس Color-SPECK

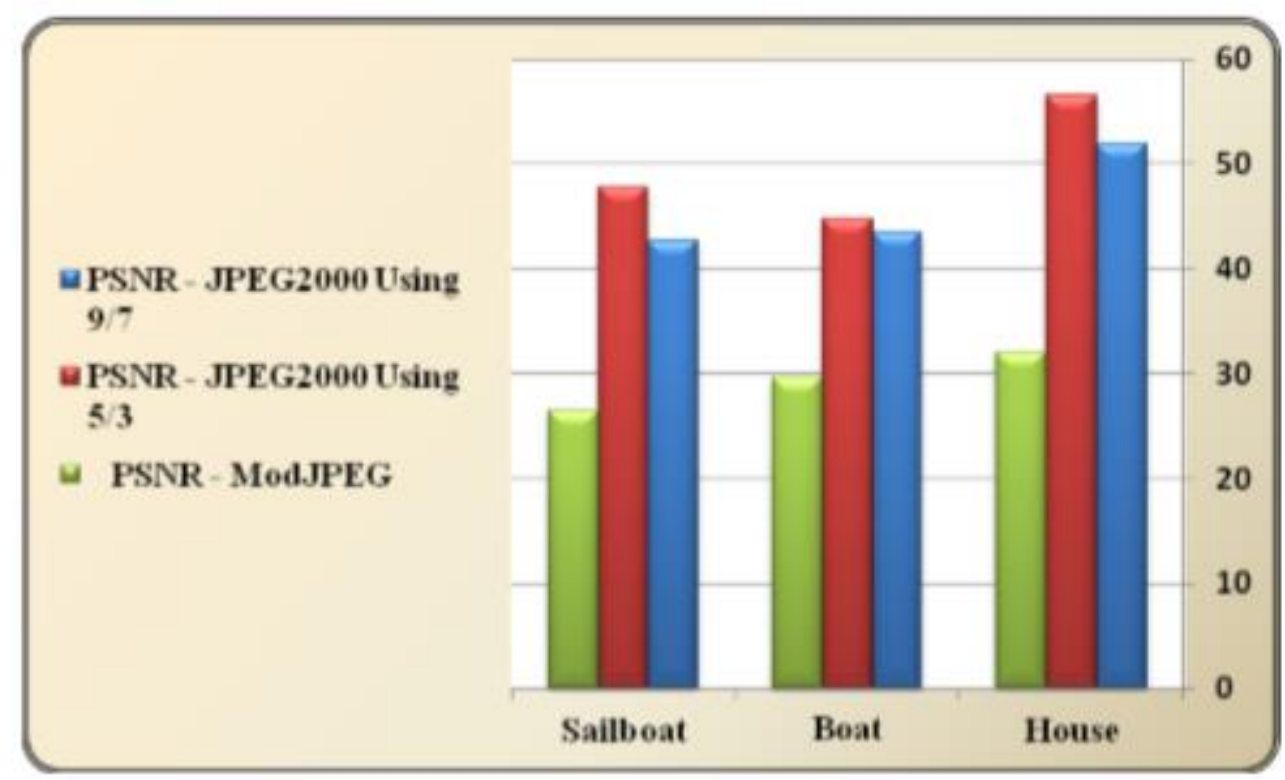

الثكل(14): مخطط مقارنة تطبيق الخوارزمية المقترحة لـ JPEG2000 باستخدام المرشحين مع خوارزمية ModJPEG للصور الملونة باستخدام المقياس PSNR. 


\section{المصادر}

[1] James Rosenthal, "JPEG Image Compression Using an FPGA",M.Sc Thesis, Electrical and Computer Engineering, University of California, Santa Barbara, December 2006.

[2] Arun Kumar P S, "Implementation of Image Compression Algorithm using Verilog with Area, Power and Timing Constraints", M.Sc Thesis, Master of Technology in VLSI Design and Embedded System, Department of Electronics and Communication Engineering National Institute of Technology, Rourkela, 2009.

[3] Athanassios N. Skodras and Touradj Ebrahimi, "JPEG2000 Image Coding System Theory and Applications", IEEE, 2010.

[4] Tinku Acharya, Ping-Sing Tsai, "JPEG2000 Standard for Image Compression Concepts, Algorithms and VLSI Architectures", p.cm., A Wiley-Interscience publication, T. Acharya Department of Engineering Arizona State University Tempe, Arizona, P. Sing Department of Computer Science The University of Texas-Pan American Edin burg, Texas, 2005.

[5] Krishna Raj, Vedvrat, "A Study of VLSI Architectures for 2-D Discrete Wavelet Transform", IEEE, 2010. 